\title{
Star $p$-hub center problem and star $p$-hub median problem with bounded path lengths
}

\author{
Hande Yaman ${ }^{a, *}$, Sourour Elloumi ${ }^{b}$ \\ a Bilkent University, Department of Industrial Engineering, Bilkent 06800 Ankara, Turkey \\ ${ }^{\mathrm{b}}$ ENSIIE-CEDRIC, 1 square de la résistance, 91025 Evry, France
}

\section{A R T I C L E I N F O}

Available online 16 February 2012

Keywords:

$p$-hub center

$p$-hub median

Star/star network

Path length constraints

Service quality

\begin{abstract}
A B S T R A C T
We consider two problems that arise in designing two-level star networks taking into account service quality considerations. Given a set of nodes with pairwise traffic demand and a central hub, we select $p$ hubs and connect them to the central hub with direct links and then we connect each nonhub node to a hub. This results in a star/star network. In the first problem, called the Star $p$-hub Center Problem, we would like to minimize the length of the longest path in the resulting network. In the second problem, Star $p$-hub Median Problem with Bounded Path Lengths, the aim is to minimize the total routing cost subject to upper bound constraints on the path lengths. We propose formulations for these problems and report the outcomes of a computational study where we compare the performances of our formulations.
\end{abstract}

(c) 2012 Elsevier Ltd. All rights reserved.

\section{Introduction}

In this paper, we consider the problem of designing a two level telecommunications network with service quality considerations. We are given a set of users or demand nodes and each of these nodes wants to communicate with all others. A fixed central hub is given and $p$ additional hubs should be chosen among the user nodes. Then each hub is connected by direct links to the central hub and each of the remaining nodes is connected directly to exactly one hub. The resulting network is a two level star/star network, where the network connecting the hub nodes to the central hub, called the backbone network, is a star, and each of the networks connecting user nodes to a hub node, called an access network, is a star.

We define two separate but related design problems for star/star networks. These problems are different from those existing in the literature as they incorporate a measure of service quality. First observe that in a star/star network, there exists a single simple path between any pair of demand nodes. If two demand nodes are connected to the same hub, then his path starts at the origin, goes directly to the hub, and then ends at the destination. If these nodes are connected to two different hubs, then the path starts at the origin, goes to the hub of the origin, then to the central hub, then to the hub of the destination, and ends at the destination. The length of

\footnotetext{
* Corresponding author.

E-mail addresses: hyaman@bilkent.edu.tr (H. Yaman), sourour.elloumi@ensiie.fr (S. Elloumi).
}

the path connecting these two nodes is taken as a measure of the quality of service for this pair of nodes.

In our first problem, we are interested in optimizing the poorest service quality in the network. Hence, our aim is to select the location of hubs and assign the remaining nodes to hubs in such a way that the longest path between two distinct nodes in the resulting network has the smallest possible value. In other words, we would like to minimize the maximum length of the path connecting any pair of distinct demand nodes. We call this problem Star $p$-hub Center Problem and abbreviate with SpHCP.

In our second problem, we also incorporate the cost of routing into the design process. Our aim is to find a network such that the total cost of routing in the network is minimum and the length of the path connecting any pair of distinct nodes does not exceed a predetermined value. This yields a solution with a given level of service quality and minimum cost. We call this problem Star p-hub Median Problem with Bounded Path Lengths and abbreviate with SpHMP-BP. If there is no limit on the path lengths and the cost of routing the traffic between the hubs and the central hub is null, then our problem is equivalent to the problem of locating $p$ hubs and allocating the remaining nodes to these hubs to minimize the total cost of allocation. Hence the $p$-median problem is a special case of our problem. As the $p$-median problem is NP-hard, SpHMP-BP is also NP-hard (for reviews on facility location problems, we refer the reader to, e.g., Cornuéjols et al. [9,10], Krarup and Pruzan [25], Labbé et al. [26] and Sridharan [37]).

To the best of our knowledge, these problems have not been studied before. Here we first review the literature on other 
star/star network design problems. The problem of minimizing the cost of establishing links and installing hubs where the traffic requirements are only from and to a central hub is studied by Gavish [17]. Here nodes are connected to the hubs via multidrop links and hubs are connected to a central unit through a star network. Different types of links with different costs and capacities are available. The problem of designing a star/star satellite communication network is studied by Helme and Magnanti [20]. Each hub has a local switch and an earth station. Nodes assigned to the same hub use the local switch and nodes assigned to different hubs use the earth station and the central hub to communicate. The problem is to choose the location of hubs and assign nodes to hubs to minimize the cost of installing hubs, connecting nodes to hubs, and using the capacities of the earth stations and the local switches. A quadratic formulation and a linearization are proposed by the authors and computational results with a branch and bound algorithm and greedy heuristics are reported. Chardaire et al. [6] present two integer programming formulations and a simulated annealing algorithm for the design of a network with two levels of hubs, i.e., each terminal is connected to a first level hub which is connected to a second level hub which is connected to a central unit. Here, traffic flows are not considered and fixed costs of connections and of installing facilities are minimized. Labbé and Yaman [28] study the problem of designing a star/star network with minimum routing cost. They do not fix the number of hubs in advance, rather they include the fixed cost of locating hubs in the total cost. The authors present formulations, polyhedral results, a branch and cut algorithm, and a Lagrangian relaxation heuristic. Yaman [39] studies a closely related problem where the aim is to locate $p$ hubs and assign the remaining nodes to hubs in order to minimize the total cost of installing capacitated links. A heuristic based on Lagrangian relaxation is proposed and computational results are given. Contreras et al. [7,8] study the problem of designing a tree/star network.

Classical hub location problems assume that hubs are connected by a complete network and the aim is to minimize the total cost of routing in the network. Hub locations problems are classified into two classes with respect to the way demand nodes are assigned to hub nodes. If a demand node can be served by several hub nodes, the problem is classified as multi-allocation and if each demand node can be served by a single hub, the problem is classified as single allocation. As both problems we consider in this paper are single allocation problems, we limit our review to this class of hub location problems. We refer the reader to Campbell et al. [5] and Alumur and Kara [1] for two recent surveys. Different formulations for hub location problems with a fixed number of hubs ( $p$-hub median problems) or with fixed costs for installing hubs (hub location with fixed costs) have been proposed in the literature (see, e.g., OKelly [31], Campbell [3], Skorin-Kapov et al. [35], Ernst and Krishnamoorthy [13], Sohn and Park [36], Ebery [12], Labbé et al. [29], and Correia et al. [11]). Ernst and Krishnamoorthy [14] present a branch and bound algorithm and an exact method based on shortest paths for the case where the number of hubs is fixed. A Lagrangian relaxation heuristic is given in Pirkul and Schilling [34]. Labbé and Yaman [27] and Labbé et al. [29] study the polyhedral properties of hub location problems.

The closest problem to our first problem SpHCP is the so-called $p$-hub center problem, which aims at minimizing the maximum transportation cost. The $p$-hub center problem is introduced by O'Kelly and Miller [32] and Campbell [3]. Campbell [3], Kara and Tansel [22], and Ernst et al. [15] give integer programming formulations, Meyer et al. [30] present a 2-phase exact algorithm, Pamuk and Sepil [33] propose a heuristic, and Juette et al. [21] give a polyhedral analysis for this problem.
A related problem is the problem of minimizing the number of hubs under the constraint of serving each pair of demand nodes within a predetermined value. This problem is called the hub covering problem. Different formulations for this problem are proposed by Kara and Tansel [23], Wagner [38], and Ernst et al. [16]. Hamacher and Meyer [19] propose an algorithm to solve the $p$-hub center problem by solving a series of hub covering problems.

There is also recent work on the problem of minimizing cost subject to a quality measure. See, for instance, Alumur et al. [2], Campbell [4], Yaman [40,41], Yaman et al. [42,43]. Our second problem SpHMP-BP belongs to this class and has features of both the $p$-hub median problem and the hub covering problem. The path length constraints are covering type constraints and are used in the hub covering problem. But in the hub covering problem, the objective is to minimize the number of hubs, whereas the objective of SpHMP-BP is to minimize the total routing cost in the network, and this is the same as the objective of the $p$-hub median problem.

In this paper, we propose formulations for SpHCP and SpHMP$B P$ and discuss the outcomes of a computational study where we compare the performances of these formulations. Our formulations model the routes between origin-destination pairs using the fact that the hub network has a star structure.

The rest of the paper is organized as follows. In Section 2, we formally define the problem $S p H C P$, prove that it is NP-hard, and present two integer programming formulations. Section 3 is devoted to the study of formulations for the SpHMP-BP. A preprocessing algorithm is also given in this section. We test our formulations from a computational point of view in Section 4 and discuss the results. We conclude the paper in Section 5.

\section{Star $p$-hub center problem}

In this section, we formally define our first problem, SpHCP, prove that it is NP-hard, and propose mixed integer programming formulations. We first give the notation. Let $I$ be the set of demand nodes and 0 be the central hub. Let $d_{i j}$ denote the distance from node $i \in I \cup\{0\}$ to node $j \in I \cup\{0\}$. We assume that $d_{i j}=d_{j i} \geq 0$ for all $i, j \in I \cup\{0\}, d_{i i}=0$ for all $i \in I \cup\{0\}$, and that the triangle inequality is satisfied, i.e., $d_{i j}+d_{j k} \geq d_{i k}$ for all $i, j, k \in I \cup\{0\}$.

The problem $\mathrm{SpHCP}$ is to locate $p$ hubs and assign each nonhub node to a hub node such that the maximum path length over all pairs of nodes is minimized. In the sequel, we assume that $p \geq 2$.

We define $x_{i j}$ to be 1 if node $i \in I$ is assigned to hub node $j \in I$ and to be 0 otherwise. If a hub is located at node $i$ then $x_{i i}$ is 1 . The variable $\beta_{\min }$ is the length of the longest path between origindestination pairs in the resulting star/star network. Using these variables, SpHCP can be formulated as follows:

$\min \beta_{\min }$

s.t. $\sum_{j \in I} x_{i j}=1 \quad \forall i \in I$

$\sum_{j \in I} x_{j j}=p$

$x_{i j} \leq x_{j j} \quad \forall i, j \in I: i \neq j$

$\left(d_{i j}+d_{0 j}\right) x_{i j}+\left(d_{0 l}+d_{l m}\right) x_{m l} \leq \beta_{\min } \quad \forall i, m, j, l \in I: i<m, j \neq l$

$d_{i j} x_{i j}+d_{m j} x_{m j} \leq \beta_{\min } \quad \forall i, m, j \in I: i<m$

$x_{i j} \in\{0,1\} \quad \forall i, j \in I$. 
Here, constraints (2) and (7) ensure that each demand node is assigned to exactly one hub node. The number of hubs is equal to $p$ due to constraint (3). Constraints (4) ensure that nodes can only be assigned to hub nodes.

We assume that all pairs of nodes need to communicate. If node $i$ is assigned to hub $j$ and node $m$ is assigned to hub $l$ different from hub $j$, then the length of the path between nodes $i$ and $m$ is equal to $d_{i j}+d_{0 j}+d_{0 l}+d_{l m}$. Constraints (5) state that $\beta_{\text {min }}$ is at least as large as the length of this path. If nodes $i$ and $m$ are assigned to the same hub $j$, then the length of the path between these nodes is equal to $d_{i j}+d_{m j}$ and due to constraints (6), $\beta_{\text {min }}$ cannot be smaller than the length of this path. As we minimize $\beta_{\min }$, in an optimal solution, the value of $\beta_{\min }$ is equal to the length of the longest simple path in the resulting star/star network.

Note that we have a major assumption here, we assume that we know the location of the central hub. If, on the contrary, we are to decide on the location of the central hub, then SpHCP can be solved for each possible location. It is also possible to incorporate the decision on the location of the central hub into our model. We define $y_{k}$ to be 1 if the central hub is located at node $k \in I$ and to be 0 otherwise. Then we add constraints $\sum_{k \in I} y_{k}=1, x_{k k} \geq y_{k}$ and $y_{k} \in\{0,1\}$ for all $k \in I$, and change constraints (5) with

$$
\begin{aligned}
& \left(d_{i j}+d_{j k}\right) x_{i j}+\left(d_{k l}+d_{l m}\right) x_{m l} \leq \beta_{\min }+M\left(1-y_{k}\right) \\
& \quad \forall i, m, j, l, k \in I: i<m, j \neq l
\end{aligned}
$$

where $M$ is a large number.

Before presenting alternative mixed integer programming formulations for $S p H C P$, we first prove that the problem is NP-hard.

\section{Theorem 1. The problem SpHCP is NP-hard.}

Proof. We define the decision version of $\mathrm{SpHCP}$ as follows. Does there exist a feasible solution to $\operatorname{SpHCP}$ with $\beta_{\min }$ less than or equal to a given positive number $K$ ? This problem is in NP. Next we show that the decision version of the unweighted vertex $p$-center problem is polynomial time reducible to the decision version of $S p H C P$. Here we use the ideas developed in Ernst et al. [15].

The decision version of the unweighted vertex $p$-center problem is defined as follows. Given a network $G=(N, E)$ with nonnegative edge lengths $c_{i j}$ for $\{i, j\} \in E$ and a positive number $K^{\prime}$, does there exist a subset $M$ of $N$ of cardinality $p$ such that $\min _{j \in M:\{i, j\} \in E} c_{i j} \leq K^{\prime}$ for all $i \in N$ ? This problem is NP-complete (see Kariv and Hakimi [24]).

For a given instance of the unweighted vertex $p$-center problem, let $I=N \cup N^{\prime}$ where $N^{\prime}$ is a copy of $N, d_{i j}=d_{i^{\prime} j}=d_{i j^{\prime}}=d_{i^{\prime} j^{\prime}}=c_{i j}$ if $\{i, j\} \in E, \quad d_{i i}=d_{i^{\prime} i}=d_{i i^{\prime}}=d_{i^{\prime} i^{\prime}}=0$ for all $i \in N$, and $d_{i j}=d_{i^{\prime} j}=d_{i j^{\prime}}=$ $d_{i^{\prime} j^{\prime}}=\infty$ for all $i, j \in N$ such that $i \neq j$ and $\{i, j\} \notin E$. Also let $d_{0 i}=d_{i 0}=d_{i^{\prime} 0}=d_{0 i^{\prime}}=0$ for all $i \in N$ and $K=2 K^{\prime}$. As a node and its copy have the same distances to all other nodes and the distance between them is zero, we know that if there exists a feasible solution to $\operatorname{SpHCP}$ with $\beta_{\min }$ less than or equal to $K$, then there exists such a solution where all $p$ hubs are chosen from the set $N, i^{\prime}$ is assigned to $i$ if $i$ is a hub and it is assigned to the same hub as $i$ if $i$ is not a hub, for all nodes $i$ in $N$ [15].

In a feasible solution for $S p H C P$, the length of the path between two nodes $i$ and $m$ is equal to $d_{i h(i)}+d_{h(m) m}$ where $h(i)$ and $h(m)$ are the hubs of $i$ and $m$, respectively. Now, if $i$ is the node whose distance to its hub is the largest, then the longest path in the network is from node $i$ to its copy $i^{\prime}$ and its length is equal to $2 d_{i h(i)}$. Hence, there exists a solution to the decision version of SpHCP if and only if there exists a solution to the decision version of the unweighted vertex $p$-center problem.
Now we proceed with our discussion of formulations. Let $n=|I|$. The above formulation uses $O\left(n^{2}\right) 0-1$ variables and has $O\left(n^{4}\right)$ constraints. Below, we first strengthen this formulation and then use auxiliary variables to decrease the number of constraints to $O\left(n^{2}\right)$. Let $X$ be the set of feasible solutions to the above formulation, i.e., $X=\left\{x \in\{0,1\}^{n^{2}}: x\right.$ satisfies (2)-(7) $\}$.

Proposition 1. For $i, m, j, l \in I$ such that $i<m$ and $j \neq l$, the inequality

$d_{i j} x_{i j}+d_{0 j} x_{j j}+d_{m l} x_{m l}+d_{0 l} x_{l l} \leq \beta_{\min }$

is valid for $X$ and implies inequality (5).

Proof. Let $x \in X$. If $x_{i j}=1$ and $x_{m l}=1$, then the left hand side of inequality (8) is the same as the one of (5) since $x_{j j}=1$ and $x_{l l}=1$. If $x_{i j}=1$ and $x_{m l}=0$, then there are two possible cases. If $x_{l l}=1$, then the left hand side of inequality (8) is equal to the length of the path between nodes $i$ and $l$ and is a lower bound for $\beta_{\text {min }}$. If $x_{l l}=0$, then $d_{i j}+d_{0 j}$ is a lower bound for the length of a path between node $i$ and any node that is selected as a hub node. If $x_{i j}=0$ and $x_{m l}=1$, we have similar cases. Finally, if both $x_{i j}=0$ and $x_{m l}=0$, then if $x_{j j}=1$ and $x_{l l}=1$, the left hand side of inequality (8) is equal to the length of the path between hubs $j$ and $l$. If $x_{j j}=1$ and $x_{l l}=0$, then $d_{0 j}$ is a lower bound for the length of a path between hub $j$ and any node that is selected as a hub. The case with $x_{j j}=0$ and $x_{l l}=1$ is similar and the case with $x_{j j}=0$ and $x_{l l}=0$ is easy. Hence, we can conclude that $x$ satisfies inequality (8). Inequality (8) implies inequality (5) since $x_{j j} \geq x_{i j}$ and $x_{l l} \geq x_{m l}$ due to constraints (4).

Hence we obtain a stronger formulation by replacing constraints (5) with (8). The resulting formulation can be further strengthened by replacing constraints (6) with the following set of inequalities

$\sum_{j \in I}\left(d_{i j} x_{i j}+d_{m j} x_{m j}\right) \leq \beta_{\min } \quad \forall i, m \in I: i<m$

These inequalities are valid since if $x_{i j}=x_{m j}=1$ for some $j \in I$, then the left hand side of (9) is equal to the left hand side of constraint (6) and if $x_{i j}=x_{m l}=1$ for two distinct hubs $j$ and $l$ in $I$, then the left hand side of (9) is less than or equal to the one of (8) for this choice of $j$ and $l$. Using inequalities (9) instead of (6) has also the advantage of decreasing the number of constraints.

Using the above results, we strengthen the starting formulation by replacing constraints (5) with (8) and constraints (6) with (9). Next we give another formulation that has the same strength as this one but has $O\left(n^{2}\right)$ constraints. We define auxiliary variables here. For $j \in I$, let $T_{j}$ be the length of the longest path from nodes assigned to $j$ to node $j$.

We replace constraints (8) with

$T_{j} \geq d_{i j} x_{i j} \quad \forall i, j \in I$

$T_{j}+d_{0 j} x_{j j}+T_{l}+d_{0 l} x_{l l} \leq \beta_{\min } \quad \forall j, l \in I: j<l$

It is easy to observe that the two formulations yield the same linear programming bound. Ernst et al. [15] use similar variables in their radius formulation for the $p$-hub center problem.

We call the above model $S p H C P-1$, i.e, minimizing $\beta_{\text {min }}$ subject to constraints (2)-(4), (7), and (9)-(11).

An alternative way to model the same problem is to use nonlinear constraints

$\sum_{j \in I}\left(d_{i j}+d_{0 j}\right) x_{i j}+\sum_{j \in I}\left(d_{0 j}+d_{m j}\right) x_{m j}-2 \sum_{j \in I} d_{0 j} x_{i j} x_{m j} \leq \beta_{\text {min }}$

$\forall i, m \in I: i<m$

instead of the system (9)-(11). Here if node $i$ is assigned to hub $j$ and node $m$ is assigned to hub $l$ different from $j$, then the length of 
the path between nodes $i$ and $m$ is equal to $d_{i j}+d_{0 j}+d_{0 l}+d_{m l}$. If nodes $i$ and $m$ are assigned to the same hub $j$, then the length of the path between these nodes is equal to $d_{i j}+d_{m j}$ as $x_{i j} x_{m j}=1$.

To linearize these constraints, we define additional variables. First note that for $i \in I$ and $j \in I$, we have $x_{i j}\left(1-x_{j j}\right)=0$ since if $x_{i j}=1$ then $x_{j j}=1$. Also for $j \in I$ and $m \in I$, we have $x_{j j}\left(1-x_{m j}\right)=x_{j j}-x_{m j}$ since $x_{j j} x_{m j}=x_{m j}$. Now let $z_{i m j}=x_{i j} x_{m j}$ for all $\left.\left.j \in I, i \in I \backslash j\right\}, m \in I \backslash j\right\}$ with $i<m$. We can replace the nonlinear constraint (12) with its linear counterpart

$$
\sum_{j \in I}\left(d_{i j}+d_{0 j}\right) x_{i j}+\sum_{j \in I}\left(d_{0 j}+d_{j m}\right) x_{m j}-\sum_{j \in \backslash \backslash i, m\}} 2 d_{0 j} z_{i m j}-2 d_{0 i} x_{m i}-2 d_{0 m} x_{i m} \leq \beta_{\min }
$$$$
\forall i, m \in I: i<m
$$

and add constraints

$$
\begin{aligned}
& z_{i m j} \leq x_{i j} \quad \forall j, i, m \in I: i<m, i \neq j, m \neq j \\
& z_{i m j} \leq x_{m j} \quad \forall j, i, m \in I: i<m, i \neq j, m \neq j
\end{aligned}
$$

Note here that we do not need to use constraints $z_{i m j} \geq x_{i j}+x_{m j}-1$ and $z_{i m j} \geq 0$. As the aim of the problem is to minimize $\beta_{\min }$, there exists an optimal solution to the problem where $z_{i m j}=\min \left\{x_{i j}, x_{m j}\right\}$ for all $j, i, m \in I$ such that $i<m, i \neq j$, and $m \neq j$.

Let $S p H C P-2$ be this new model, i.e, minimizing $\beta_{\min }$ subject to constraints (2)-(4), (7), and (13)-(15).

To conclude this section, we note that $S p H C P-1$ has $O\left(n^{2}\right)$ variables and $O\left(n^{2}\right)$ constraints and $S p H C P-2$ has $O\left(n^{3}\right)$ variables and $O\left(n^{3}\right)$ constraints. We compare the computational performances of these two formulations in Section 4.

\section{Star $p$-hub median problem with bounded path lengths}

In this section, we propose models for $S p H M P-B P$. We first introduce more notation. Let $t_{i m}$ denote the amount of traffic to be routed from node $i \in I$ to node $m \in I$. As the traffic from a node to itself does not travel on the arcs of the network, we assume that $t_{i i}=0$ for all $i \in I$. We denote the cost of routing a unit traffic from node $i \in I \cup\{0\}$ to node $j \in I \cup\{0\}$ by $f_{i j}$. We assume that $f_{j j}=0$ for all $j \in I$. Let $c_{i j}=f_{i j} \sum_{m \in I} t_{i m}+f_{j i} \sum_{m \in I} t_{m i}$ for $i \in I$ and $j \in I \backslash\{i\}$ and $c_{j j}=0$ for $j \in I$.

The problem SpHMP-BP is to locate $p$ hubs and assign each nonhub node to a hub node such that the length of the simple path between any pair of nodes does not exceed the bound $\beta$. The aim is to minimize the total cost of routing. We again assume that $p \geq 2$.

We first propose a nonlinear model using the $0-1$ variables $x_{i j}$ 's and the auxiliary variables $T_{j}$ 's.

$\min \sum_{i \in I j} \sum_{j \in \backslash \backslash i\}} c_{i j} x_{i j}+\sum_{j \in I} \sum_{i \in I} \sum_{m \in I\{i\}}\left(f_{j 0} t_{i m}+f_{0 j} t_{m i}\right) x_{i j}\left(1-x_{m j}\right)$

s.t. $\quad(2)-(4),(7),(10)$

$$
T_{j}+d_{0 j} x_{j j}+T_{l}+d_{0 l} x_{l l} \leq \beta \quad \forall j, l \in I: j<l
$$

$$
\sum_{j \in I}\left(d_{i j} x_{i j}+d_{m j} x_{m j}\right) \leq \beta \quad \forall i, m \in I: i<m
$$

Constraints (17) and (18) are the same as those used for modeling SpHCP. The only difference here is that $\beta$ is a parameter of the problem.

We explain the objective function in more detail. If node $i$ is assigned to hub $j$, then the total traffic traveling from $i$ to $j$ is equal to $\sum_{m \in I} t_{i m}$ and the total traffic traveling from $j$ to $i$ is equal to $\sum_{m \in I} t_{m i}$. The cost of routing this traffic in both ways is denoted by $c_{i j}$. The traffic traveling from hub $j$ to the central hub is equal to the total traffic from the nodes that are assigned to hub $j$ to the nodes that are assigned to other hubs, i.e., $\sum_{i \in I} \sum_{m \in \backslash \backslash i\}} t_{i m} x_{i j}\left(1-x_{m j}\right)$. Similarly, the total traffic traveling from the central hub to hub $j$ is equal to $\sum_{i \in I} \sum_{m \in I \backslash i\}} t_{m i} x_{i j}\left(1-x_{m j}\right)$. The objective function (16) is the total cost of routing the traffic in the network.

Next, we provide a linear $0-1$ model for our problem using the variables $z_{i m j}$ 's defined in the previous section. First, note that, as we have $x_{i j}\left(1-x_{j j}\right)=0$ for $i \in I$ and $j \in I$ and we have $x_{j j}\left(1-x_{m j}\right)=$ $x_{j j}-x_{m j}$ for $j \in I$ and $m \in I$, the objective function (16) can be rewritten as:

$$
\begin{aligned}
\min & \sum_{i \in I j \in I \backslash i\}} \sum_{j}\left(c_{i j}-\left(f_{j 0} t_{j i}+f_{0 j} t_{i j}\right)+\sum_{m \in \Lambda \backslash i, j\}}\left(f_{j 0} t_{i m}+f_{0 j} t_{m i}\right)\right) x_{i j} \\
& +\sum_{j \in I} \sum_{i \in I}\left(f_{j 0} t_{j i}+f_{0 j} t_{i j}\right) x_{j j} \\
& -\sum_{j \in I i \in \backslash \backslash j\} m \in I \backslash i, j\}} \sum_{j 0}\left(f_{i m} t_{i j}+f_{0 j} t_{m i}\right) x_{i j} x_{m j} .
\end{aligned}
$$

Now our model can be linearized by replacing the objective function with

$$
\begin{aligned}
\min & \sum_{i \in I j \in I \backslash i\}} \sum_{j}\left(c_{i j}-\left(f_{j 0} t_{j i}+f_{0 j} t_{i j}\right)+\sum_{m \in \Lambda \backslash i, j\}}\left(f_{j 0} t_{i m}+f_{0 j} t_{m i}\right)\right) x_{i j} \\
& +\sum_{j \in I} \sum_{i \in I}\left(f_{j 0} t_{j i}+f_{0 j} t_{i j}\right) x_{j j} \\
& -\sum_{j \in I} \sum_{i \in \Lambda \backslash j\} m \in} \sum_{\backslash \backslash j\}: i<m}\left(f_{j 0} t_{i m}+f_{0 j} t_{m i}+f_{j 0} t_{m i}+f_{0 j} t_{i m}\right) z_{i m j}
\end{aligned}
$$

and adding the constraints (14) and (15).

We call the resulting model SpHMP-BP-1. Our second formulation $S p H M P-B P-2$ is obtained by dropping the auxiliary variables $T_{j}$ 's and replacing constraints (10), (17), and (18) with constraints $\sum_{j \in I}\left(d_{i j}+d_{0 j}\right) x_{i j}+\sum_{j \in I}\left(d_{m j}+d_{0 j}\right) x_{m j}-\sum_{j \in I \backslash i, m\}} 2 d_{0 j} z_{i m j}-2 d_{0 i} x_{m i}-2 d_{0 m} x_{i m} \leq \beta$

Finally, we propose a model which imposes conflicts due to path lengths using clique inequalities. Wagner [38] proposes a similar formulation for the hub covering problem. For $i \in I, j \in I$, and $m \in I \backslash\{i, j\}$, let $A_{i j m}$ be the set of nodes to which node $m$ cannot be assigned when node $i$ is assigned to hub $j$. Algorithm 1 computes sets $A_{i j m}$. Suppose that node $i$ is assigned to hub $j$. Then, if $i \neq j, m$ cannot be assigned to node $i$. If $d_{i j}+d_{m j}>\beta$, then node $m$ cannot be assigned to hub $j$ together with node $i$. If $d_{i j}+d_{m j} \leq \beta$ then we may still have a conflict as when $i$ and $m$ are assigned to hub $j$, we need $p-1$ more nodes as hubs. Observe that if node $k \neq i, j, m$ is selected as a hub node, the length of the path between $k$ and $i$ is equal to $d_{i j}+d_{0 j}+d_{0 k}$ and the length of the path between $k$ and $m$ is equal to $d_{m j}+d_{0 j}+d_{0 k}$ and both these quantities should not exceed the bound $\beta$. Hence if $\left|\left\{k \in J\{i, j, m\}: \max \left\{d_{i j}, d_{m j}\right\}+d_{0 j}+d_{0 k} \leq \beta\right\}\right| \leq p-2$, then nodes $i$ and $m$ cannot be assigned to hub $j$ at the same time.

Now consider a node $l \neq i, j$. If $d_{i j}+d_{0 j}+d_{0 l}+d_{m l}>\beta$, then assigning node $m$ to hub $l$ results in a path of length longer than $\beta$. If $d_{i j}+d_{0 j}+d_{0 l}+d_{m l} \leq \beta$ but the cardinality of the set $\left\{k \in J\{i, j, m, l\}: d_{i j}+d_{0 j}+d_{0 k} \leq \beta\right.$ and $\left.d_{m l}+d_{0 l}+d_{0 k} \leq \beta\right\}$ is less than $p-2$, then if $m$ is assigned to hub $l$, then it is not possible to find $p-2$ nodes to install hubs together with $j$ and $l$. Hence assigning $i$ to $j$ and $m$ to $l$ at the same time causes infeasibility if $p \geq 3$.

Algorithm 1. Computation of sets $A_{i j m}$ for $i, j, m \in I$ such that $m \neq i, j$.

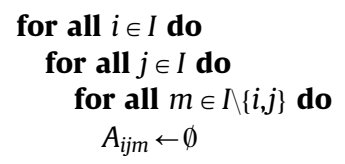




$$
\begin{gathered}
\text { if } i \neq j \text { then } \\
A_{i j m} \leftarrow\{i\} \\
\text { if } d_{i j}+d_{m j}>\beta \text { or } \mid\{k \in I \backslash i, j, m\}: \\
\left.\max \left\{d_{i j}, d_{m j}\right\}+d_{0 j}+d_{0 k} \leq \beta\right\} \mid \leq p-2 \text { then } \\
A_{i j m} \leftarrow A_{i j m} \cup\{j\} \\
\text { for all } l \in I \backslash\{i, j\} \text { do } \\
\text { if } d_{i j}+d_{0 j}+d_{0 l}+d_{m l}>\beta \text { or }(p \geq 3 \text { and } \\
\left.\mid\left\{k \in I \backslash\{i, j, m, l\}: d_{i j}+d_{0 j}+d_{0 k} \leq \beta \text { and } d_{m l}+d_{0 l}+d_{0 k} \leq \beta\right\} \mid \leq p-3\right)
\end{gathered}
$$

then

$$
A_{i j m} \leftarrow A_{i j m} \cup\{l\}
$$

For $i \in I, j \in I$, and $m \in I \backslash\{i, j\}$ such that $\left|A_{i j m}\right| \geq 2$ or $\left|A_{i j m}\right| \geq 1$ and $i=j$, the clique inequality

$x_{i j}+\sum_{l \in A_{i j m}} x_{m l} \leq 1$

should be satisfied by all feasible solutions. Moreover, any 0-1 vector $x$ which satisfies clique inequalities (20) does not violate the path length restrictions.

Let SpHMP-BP-3 be the formulation obtained from SpHMP-BP-2 by replacing constraints (19) with (20).

Before concluding this section, we give simple ideas of preprocessing which can be applied to all three formulations for SpHMP-BP. First we give a proposition, which can help to detect infeasibility.

Proposition 2. If there exists a node $j \in I$ such that $\mid\{l \in I \backslash j\}$ : $\left.d_{0 j}+d_{0 l} \leq \beta\right\} \mid \leq p-2$ then SpHMP-BP is infeasible.

Proof. Suppose that there exists a node $j \in I$ such that $\mid\{l \in I \backslash j\}$ : $\left.d_{0 j}+d_{0 l} \leq \beta\right\} \mid \leq p-2$ and that the problem has a feasible solution. Let node $k$ be the hub to which node $j$ is assigned to and $J$ be the set of hubs in this solution. Then $d_{j k}+d_{0 k}+d_{0 l} \leq \beta$ for all $l \in J\{k\}$. Hence there exist at least $p-1$ nodes different from $j$ such that $d_{j k}+d_{0 k}+d_{0 l} \leq \beta$. As the distances satisfy the triangle inequality, $d_{0 j}+d_{0 l} \leq d_{j k}+d_{0 k}+d_{0 l}$. This is in conflict with $j$ being a node such that $\left.\mid\{l \in I \backslash j\}: d_{0 j}+d_{0 l} \leq \beta\right\} \mid \leq p-2$.

If we cannot detect infeasibility, we can use the following ideas to fix the values of some of the variables.

Proposition 3. Let $i \in I$ and $j \in I \backslash\{i\}$. If $d_{i j}+d_{0 j}>\beta$ or $\mid\{l \in I \backslash\{i, j\}$ : $\left.d_{i j}+d_{0 j}+d_{0 l} \leq \beta\right\} \mid \leq p-2$, then all feasible solutions satisfy $x_{i j}=0$.

Proposition 4. Let $i, m \in I$ such that $i<m$ and $j \in I \backslash\{i, m\}$. If $d_{i j}+d_{m j}>\beta$ or $\left.\mid\{k \in I \backslash i, j, m\}: \max \left\{d_{i j}, d_{m j}\right\}+d_{0 j}+d_{0 k} \leq \beta\right\} \mid \leq p-2$, then all feasible solutions satisfy $z_{i m j}=0$.

The proofs are omitted as they are similar to the proof of Proposition 2.

Algorithm 2 uses the results of the two propositions above to fix the values of variables.

\section{Algorithm 2. Variable fixing.}

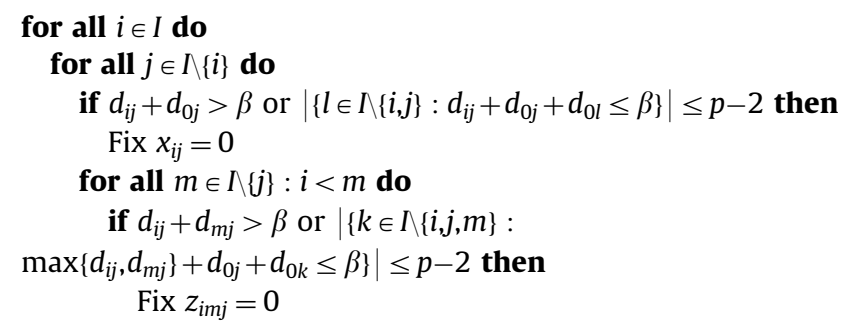

In the next section, we provide a computational study where we compare our three formulations and investigate the effect of preprocessing.

\section{Computational results}

In this section, we report the outcomes of our computational study. First, we use instances from the AP data set of Ernst and Krishnamoorthy [13]. From the coordinates of the nodes provided in the input data files, we compute distances $d_{i j}$ as the Euclidean distances divided by 100 and round to the closest integer. As the rounding may introduce violation of the triangle inequalities, we apply a correcting procedure to the distances. We fix $n$ to 50 and make $p$ take the values $2,4,6,8$, and 10 . We choose node 6 as the central hub as its coordinates are close to the center.

We have five instances of $S p H C P$ with different $p$ values. We compute $\beta_{\min }$ by solving our two formulations SpHCP-1 and SpHCP-2.

For $S p H M P-B P$, we need additional data. We use the amounts of traffic $t_{i m}$ available in the data files. We take the unit routing costs $f_{i j}$ as equal to the distances $d_{i j}$. For each choice of $p$, we take the limit on the path lengths $\beta$ equal to $\beta_{\min },\left\lceil 1.1 \times \beta_{\min }\right\rceil$, $\left\lceil 1.2 \times \beta_{\min }\right\rceil$, and $\left\lceil 1.3 \times \beta_{\min }\right\rceil$. Clearly, $\beta_{\min }$ is the smallest value of $\beta$ such that problem SpHMP-BP is feasible. We have 20 instances of the SpHMP-BP problem.

We use the mixed integer programming (MIP) solver of CPLEX 11.0 to solve all the formulations. We use the default settings of CPLEX. Our experiments are carried out on a PC with an Intel core 2 duo processor of $2.8 \mathrm{GHz}$ and $2048 \mathrm{MB}$ of RAM using a Linux operating system.

In Tables 1 and 2, we report the results that we obtained by solving the different formulations for the two problems, SpHCP and $S p H M P-B P$. In these tables, gap, nds, and cpu contain the percentage gap between the optimal value and the LP-relaxation bound, the number of nodes in the branch-and-cut tree, and the running time in seconds, respectively.

We can observe from Table 1 that the LP-relaxation bound associated with formulation $S p H C P-1$ is very poor while the bound associated with formulation $S p H C P-2$ is quite tight. However, formulation $S p H C P-1$ can solve all the instances within an average of $709 \mathrm{~s}$ but formulation $\mathrm{SpHCP}-2$ needs more than $4 \mathrm{~h}$ in average. This is due to the larger size of formulation $S p H C P-2$. For example, with $n=50$ and $p=2$ and after the MIP presolve phase, formulation SpHCP-1 has 2551 columns and 7401 rows while formulation SpHCP-2 has 60,125 columns and 118,974 rows. Hence, the MILP formulation SpHCP-2 that is built as the linearization of a quadratic formulation is very strong from the LP-bound point of view but it has the drawback of adding a huge number of columns and rows when compared to the initially linear formulation $S p H C P-1$.

Problem $S p H M P-B P$ is structurally different from $S p H C P$ since non-linearity appears also in the objective function. In both

\begin{tabular}{|c|c|c|c|c|c|c|c|c|}
\hline \multirow[t]{2}{*}{$n$} & \multirow[t]{2}{*}{$p$} & \multirow[t]{2}{*}{$\beta_{\min }$} & \multicolumn{3}{|l|}{ SpHCP-1 } & \multicolumn{3}{|l|}{ SpHCP-2 } \\
\hline & & & gap (\%) & nds & $\mathrm{cpu}$ & gap (\%) & nds & cpu \\
\hline 50 & 2 & 680 & 40.8 & 584 & 120 & 0.3 & 8 & 20,023 \\
\hline 50 & 4 & 680 & 66.7 & 10,788 & 1423 & 0.3 & 7 & 10,878 \\
\hline 50 & 6 & 680 & 68.3 & 20,721 & 977 & 0.3 & 28 & 20,162 \\
\hline 50 & 8 & 680 & 68.3 & 4964 & 519 & 0.3 & 22 & 15,384 \\
\hline 50 & 10 & 680 & 68.3 & 12,653 & 509 & 0.3 & 37 & 11,316 \\
\hline
\end{tabular}
formulations we consider, based on the $x$ and $T$ variables or based on the $x$ variables only, we had to further linearize by use of the $z$ variables. This leads to formulations with a big size. For SpHMP-BP instances, we set a time limit of $1 \mathrm{~h}$.

In Table 2, we report the results for SpHMP-BP. Here, for formulation SpHMP-BP-1, column cpu reports the running time if the

Table 1

Results for the star $p$-hub center problem SpHCP for the AP instances. 
Table 2

Results for the star $p$-hub median problem with bounded path lengths SpHMP-BP for the AP instances.

\begin{tabular}{|c|c|c|c|c|c|c|c|c|c|c|c|c|c|c|c|c|c|c|c|c|}
\hline \multirow[t]{2}{*}{$n$} & \multirow[t]{2}{*}{$p$} & \multirow[t]{2}{*}{$\beta$} & \multirow[t]{2}{*}{ opt } & \multicolumn{4}{|c|}{ SpHMP-BP-1 } & \multicolumn{4}{|c|}{ SpHMP-BP-2 } & \multicolumn{5}{|c|}{ SpHMP-BP-3 } & \multicolumn{4}{|c|}{ SpHMP-BP-3 and preproc } \\
\hline & & & & gap (\%) & nds & cpu & сpu- & gap (\%) & nds & cpu & cpu- & gap (\%) & nds & cpu & cpu- & cliques & $\mathrm{cpu}$ & cpu- & \%fix $x$ & \%fix $z$ \\
\hline 50 & 2 & 680 & $1,215,221.8$ & 25.0 & 110 & $12.4 \%$ & $21.9 \%$ & 15.8 & 0 & 1968 & $13.0 \%$ & 0.0 & 0 & 329 & 237 & 120,050 & 344 & 339 & 6 & 13 \\
\hline 50 & 2 & 748 & $997,393.3$ & 8.9 & 196 & $2.9 \%$ & $7.6 \%$ & 8.4 & 0 & 1728 & $1.1 \%$ & 0.0 & 0 & 609 & 615 & 120,035 & 136 & 136 & 3 & 8 \\
\hline 50 & 2 & 816 & $945,884.5$ & 4.0 & 39 & 953 & $1.0 \%$ & 4.0 & 3 & 837 & 994 & 0.0 & 0 & 1032 & 1008 & 119,846 & 102 & 700 & 1 & 4 \\
\hline 50 & 2 & 884 & $915,111.6$ & 0.9 & 3 & 413 & 569 & 0.9 & 3 & 367 & 300 & 0.0 & 0 & 425 & 594 & 118,942 & 451 & 650 & 0 & 2 \\
\hline 50 & 4 & 680 & $1,213,977.4$ & 25.3 & 191 & $21.7 \%$ & $24.8 \%$ & 16.8 & 0 & 2906 & $16.2 \%$ & 0.0 & 0 & 239 & 265 & 120,050 & 250 & 195 & 10 & 17 \\
\hline 50 & 4 & 748 & $994,080.7$ & 8.9 & 145 & $6.8 \%$ & $8.4 \%$ & 8.3 & 0 & 1324 & $6.4 \%$ & 0.0 & 0 & 132 & 142 & 120,050 & 135 & 133 & 6 & 11 \\
\hline 50 & 4 & 816 & $944,640.1$ & 4.2 & 476 & $2.3 \%$ & $3.3 \%$ & 4.0 & 0 & 827 & 2004 & 0.0 & 0 & 622 & 736 & 119,883 & 108 & 676 & 3 & 6 \\
\hline 50 & 4 & 884 & $911,799.0$ & 0.8 & 13 & 422 & 511 & 0.7 & 0 & 409 & 428 & 0.0 & 0 & 434 & 429 & 118,954 & 444 & 439 & 1 & 3 \\
\hline 50 & 6 & 680 & $1,214,804.8$ & 25.4 & 48 & $20.8 \%$ & $24.9 \%$ & 16.9 & 0 & $0.3 \%$ & $16.6 \%$ & 0.0 & 0 & 139 & 119 & 120,050 & 119 & 146 & 11 & 19 \\
\hline 50 & 6 & 748 & $994,080.7$ & 8.9 & 246 & $5.9 \%$ & $8.2 \%$ & 8.3 & 0 & 1364 & $4.6 \%$ & 0.0 & 0 & 124 & 125 & 120,050 & 121 & 114 & 6 & 12 \\
\hline 50 & 6 & 816 & $945,876.2$ & 4.3 & 476 & $2.5 \%$ & $3.4 \%$ & 4.2 & 17 & 1037 & $2.1 \%$ & 0.0 & 0 & 644 & 587 & 119,914 & 108 & 728 & 3 & 7 \\
\hline 50 & 6 & 884 & $911,799.0$ & 0.8 & 41 & 505 & 627 & 0.7 & 0 & 389 & 386 & 0.0 & 0 & 387 & 381 & 118,986 & 995 & 407 & 1 & 3 \\
\hline 50 & 8 & 680 & $1,220,719.8$ & 25.7 & 98 & $22.3 \%$ & $25.2 \%$ & 17.3 & 0 & 1992 & $16.9 \%$ & 0.0 & 0 & 111 & 111 & 120,050 & 113 & 103 & 14 & 23 \\
\hline 50 & 8 & 748 & $994,080.7$ & 8.8 & 67 & $7.0 \%$ & $8.4 \%$ & 8.3 & 142 & 2966 & $7.8 \%$ & 0.0 & 0 & 120 & 119 & 120,050 & 118 & 113 & 8 & 14 \\
\hline 50 & 8 & 816 & $949,764.1$ & 4.7 & 476 & $2.8 \%$ & $3.9 \%$ & 4.5 & 11 & 1089 & $2.0 \%$ & 0.1 & 3 & 309 & 247 & 119,989 & 301 & 241 & 5 & 9 \\
\hline 50 & 8 & 884 & $912,244.9$ & 0.8 & 63 & 664 & 682 & 0.7 & 0 & 361 & 372 & 0.0 & 0 & 271 & 322 & 119,122 & 506 & 418 & 2 & 4 \\
\hline 50 & 10 & 680 & $1,231,092.1$ & 26.1 & 214 & $21.2 \%$ & $25.8 \%$ & 17.8 & 14 & 3100 & $17.1 \%$ & 0.0 & 0 & 120 & 156 & 120,050 & 151 & 123 & 15 & 24 \\
\hline 50 & 10 & 748 & $994,080.7$ & 8.6 & 195 & $5.8 \%$ & $7.8 \%$ & 8.2 & 25 & 1502 & $5.7 \%$ & 0.0 & 0 & 127 & 127 & 120,050 & 125 & 133 & 9 & 14 \\
\hline 50 & 10 & 816 & $957,072.2$ & 5.1 & 1140 & $3.5 \%$ & $4.4 \%$ & 5.0 & 118 & 1676 & $4.0 \%$ & 0.5 & 5 & 401 & 329 & 119,996 & 472 & 343 & 5 & 9 \\
\hline 50 & 10 & 884 & $913,485.7$ & 0.6 & 28 & 539 & 539 & 0.6 & 3 & 383 & 348 & 0.0 & 0 & 438 & 322 & 119,166 & 418 & 536 & 2 & 4 \\
\hline
\end{tabular}

Table 3

Results for the star $p$-hub median problem with bounded path lengths SpHMP-BP for the randomly generated instances.

\begin{tabular}{|c|c|c|c|c|c|c|c|c|c|c|c|c|c|c|c|c|c|c|c|}
\hline \multirow[t]{2}{*}{$n$} & \multirow[t]{2}{*}{$p$} & \multirow[t]{2}{*}{$\beta$} & \multirow[t]{2}{*}{ opt } & \multicolumn{4}{|l|}{ SpHMP-BP-1 } & \multicolumn{4}{|l|}{ SpHMP-BP-2 } & \multicolumn{5}{|l|}{ SpHMP-BP-3 } & \multicolumn{3}{|c|}{$\begin{array}{l}\text { SpHMP-BP-3 and } \\
\text { preproc }\end{array}$} \\
\hline & & & & $l b$ & gap (\%) & nds & cpu & $l b$ & gap (\%) & nds & cpu & $l b$ & gap (\%) & nds & cpu & cliques & $\mathrm{cpu}$ & \%fix $x$ & \%fix $z$ \\
\hline 30 & 4 & 129 & 291,376 & $291,376.0$ & 0.0 & 0 & 12.0 & $291,376.0$ & 0.0 & 0 & 3 & $291,376.0$ & 0.0 & 0 & 1 & 25,230 & 1 & 33 & 54 \\
\hline 30 & 4 & 142 & 291,376 & $291,376.0$ & 0.0 & 0 & 7.1 & $291,376.0$ & 0.0 & 0 & 4 & $291,376.0$ & 0.0 & 0 & 3 & 25,230 & 2 & 23 & 41 \\
\hline 30 & 4 & 155 & 291,376 & $291,376.0$ & 0.0 & 476 & 9.8 & $291,376.0$ & 0.0 & 0 & 4 & $291,376.0$ & 0.0 & 0 & 4 & 25,230 & 4 & 16 & 29 \\
\hline 30 & 7 & 159 & 370,379 & $326,072.1$ & 12.0 & 0 & 45.7 & $326,072.1$ & 12.0 & 0 & 20 & $370,379.0$ & 0.0 & 0 & 1 & 25,230 & 0 & 48 & 65 \\
\hline 30 & 7 & 175 & 340,012 & $326,072.1$ & 4.1 & 24 & 65.6 & $326,072.1$ & 4.1 & 4 & 39 & $339,740.0$ & 0.1 & 0 & 2 & 25,230 & 1 & 37 & 53 \\
\hline 30 & 7 & 191 & 338,348 & $326,072.1$ & 3.6 & 75 & 115.8 & $326,072.1$ & 3.6 & 3 & 23 & $337,578.3$ & 0.2 & 7 & 14 & 25,230 & 13 & 23 & 37 \\
\hline 40 & 6 & 133 & 564,850 & $557,350.0$ & 1.3 & 491 & 1844.3 & $561,121.6$ & 0.7 & 5 & 110 & $564,850.0$ & 0.0 & 0 & 6 & 60,840 & 4 & 31 & 52 \\
\hline 40 & 6 & 146 & 559,897 & $557,042.7$ & 0.5 & 20 & 296.0 & $557,675.6$ & 0.4 & 5 & 113 & $559,897.0$ & 0.0 & 0 & 11 & 60,840 & 9 & 19 & 37 \\
\hline 40 & 6 & 160 & 556,985 & $556,985.0$ & 0.0 & 0 & 163.4 & $556,985.0$ & 0.0 & 0 & 82 & $556,985.0$ & 0.0 & 0 & 16 & 60,781 & 14 & 10 & 22 \\
\hline 40 & 9 & 136 & 538,428 & $534,891.3$ & 0.7 & 89 & 366.3 & $535,682.2$ & 0.5 & 0 & 78 & $538,269.7$ & 0.0 & 0 & 13 & 60,840 & 11 & 29 & 45 \\
\hline 40 & 9 & 150 & 538,428 & $534,764.5$ & 0.7 & 375 & 942.2 & $534,969.9$ & 0.6 & 17 & 157 & $537,023.6$ & 0.3 & 7 & 73 & 60,840 & 73 & 19 & 32 \\
\hline 40 & 9 & 163 & 534,692 & $534,692.0$ & 0.0 & 0 & 143.4 & $534,692.0$ & 0.0 & 0 & 67 & $534,692.0$ & 0.0 & 0 & 20 & 60,816 & 20 & 12 & 22 \\
\hline 50 & 8 & 126 & 775,232 & $749,829.8$ & 3.3 & 265 & $5.75 \%$ & $764,794.0$ & 1.3 & 13 & 972 & $775,232.0$ & 0.0 & 0 & 23 & 120,050 & 16 & 20 & 35 \\
\hline 50 & 8 & 138 & 749,727 & $749,727.0$ & 0.0 & 0 & 712.54 & $749,727.0$ & 0.0 & 0 & 578 & $749,727.0$ & 0.0 & 0 & 38 & 120,050 & 32 & 12 & 22 \\
\hline 50 & 8 & 152 & 749,727 & $749,727.0$ & 0.0 & 0 & 624.89 & $749,727.0$ & 0.0 & 0 & 611 & $749,727.0$ & 0.0 & 0 & 69 & 119,928 & 66 & 7 & 14 \\
\hline 50 & 20 & 130 & 866,108 & $855,366.5$ & 1.2 & 0 & 1399.57 & $856,154.8$ & 1.1 & 6 & 1489 & $866,108.0$ & 0.0 & 0 & 14 & 120,050 & 5 & 48 & 64 \\
\hline 50 & 20 & 143 & 855,023 & $855,023.0$ & 0.0 & 0 & 1070.49 & $855,023.0$ & 0.0 & 0 & 971 & $855,023.0$ & 0.0 & 0 & 20 & 120,050 & 12 & 36 & 50 \\
\hline 50 & 20 & 157 & 855,023 & $855,023.0$ & 0.0 & 0 & 1149.5 & $855,023.0$ & 0.0 & 0 & 976 & $855,023.0$ & 0.0 & 0 & 47 & 120,050 & 38 & 24 & 36 \\
\hline 60 & 10 & 141 & $1,294,424$ & - & - & - & - & - & - & - & - & $1,294,424.0$ & 0.0 & 0 & 53 & 208,860 & 29 & 38 & 54 \\
\hline 60 & 10 & 155 & $1,289,365$ & - & - & - & - & - & - & - & - & $1,288,538.0$ & 0.1 & 0 & 263 & 208,860 & 242 & 26 & 39 \\
\hline 60 & 10 & 170 & $1,289,365$ & - & - & - & - & - & - & - & - & $1,288,538.0$ & 0.1 & 0 & 842 & 208,860 & 671 & 17 & 27 \\
\hline 60 & 25 & 130 & $1,154,062$ & $1,110,903.9$ & 3.7 & - & - & $1,116,385.0$ & 3.3 & 24 & - & $1,154,062.0$ & 0.0 & 0 & 98 & 208,860 & 78 & 30 & 43 \\
\hline 60 & 25 & 143 & $1,121,533$ & $1,110,824.0$ & 1.0 & 30 & $0.89 \%$ & $1,110,876.8$ & 1.0 & 85 & $0.88 \%$ & $1,121,461.0$ & 0.0 & 0 & 218 & 208,860 & 203 & 21 & 31 \\
\hline 60 & 25 & 157 & $1,110,988$ & $1,110,819.4$ & 0.0 & 2 & 2894.9 & $1,110,819.4$ & 0.0 & 2 & 3089 & $1,110,819.4$ & 0.0 & 0 & 358 & 208,836 & 345 & 13 & 20 \\
\hline 70 & 8 & 135 & $1,829,673$ & - & - & - & - & - & - & - & - & $1,829,673.0$ & 0.0 & 0 & 99 & 333,270 & 48 & 41 & 63 \\
\hline 70 & 8 & 148 & $1,800,788$ & - & - & - & - & - & - & - & - & $1,800,788.0$ & 0.0 & 0 & 131 & 333,270 & 99 & 29 & 50 \\
\hline 70 & 8 & 163 & $1,800,788$ & - & - & - & - & - & - & - & - & $1,800,788.0$ & 0.0 & 0 & 224 & 333,263 & 245 & 19 & 34 \\
\hline 70 & 20 & 136 & $1,651,304$ & - & - & - & - & - & - & - & - & $1,650,919.0$ & 0.0 & 0 & 91 & 333,270 & 47 & 36 & 51 \\
\hline 70 & 20 & 150 & $1,646,541$ & - & - & - & - & - & - & - & - & $1,645,892.5$ & 0.0 & 0 & 706 & 333,270 & 673 & 25 & 37 \\
\hline 70 & 20 & 164 & $1,646,541$ & - & - & - & - & - & - & - & - & $1,644,963.7$ & 0.0 & 7 & 1529 & 333,270 & 1439 & 16 & 26 \\
\hline
\end{tabular}

branch-and-cut algorithm can prove optimality within $1 \mathrm{~h}$. If the branch-and-cut is stopped by the time limit, a percentage that represents the relative gap between the best obtained lower bound and the optimal value opt is reported in this column. To see the effect of Cplex cuts on the solution times, we also solved the same instances by disabling these cuts. We report the solution times in columns cpu-. 
With default Cplex settings, formulation SpHMP-BP-1 fails in solving 14 instances over 20 within the time limit. However, formulation SpHMP-BP-2 succeeds in solving 19 instances over the 20 within the time limit and with an average time of $1311 \mathrm{~s}$. We can observe that the average gap associated with SpHMP-BP-2 is of about $7.5 \%$ and is not much better than the $10 \%$ average gap associated with SpHMP-BP-1. However the number of nodes is significantly smaller with SpHMP-BP-2. This indicates that constraints (19) are not very strong from the LP-relaxation point of view, but they can drastically help the branch-and-cut process. In our implementation of SpHMP-BP-3, we put all the cliques computed by Algorithm 1 . The number of cliques is given in column cliques. We can observe that the obtained LP-relaxation bound is then very strong since it is equal to the optimal solution value for 18 instances over 20 . The average solution time for formulation SpHMP-BP-3 is $350 \mathrm{~s}$. Finally, we provide the results of the variable fixing procedure in Algorithm 2, applied to SpHMP$B P-3$. The LP-relaxation bound is not reported in Table 2 because we observed that it is always equal to the LP-relaxation bound of SpHMP-BP-3. However, a significant percentage of $x$ and $z$ variables are fixed and the average solution time decreases to $275 \mathrm{~s}$. We see that disabling the Cplex cuts hurts significantly the performance of the formulations SpHMP-BP-1 and SpHMP-BP-2, whereas this does not have a big effect on the performance of the formulation SpHMP-BP-3.

Finally, we use some randomly generated instances to compare the performances of formulations for SpHMP-BP. These instances are generated as follows. The nodes are generated in the plane with coordinates uniformly distributed in $[1,10,000]$. The amount of traffic $t_{i j}$ is generated uniformly in the interval $[0,9]$. The distance $d_{i j}$ is computed as the euclidean distance divided by 100 and rounded. Then the distances are corrected to make sure that they satisfy the triangle inequality. The costs $f_{i j}$ are set equal to the distances $d_{i j}$. In this experiment, we let Cplex generate its cuts. For the instances other than the one with 70 nodes and 20 hubs, we computed optimally the $\beta_{\min }$ values. For the instance, we took $\beta_{\min }$ equal to the smallest value $\beta$ for which we could compute a feasible solution. We set the first $\beta$ value equal to $\beta_{\min }$ and gradually increase.

The results are given in Table 3. The optimal values of the LP relaxations are reported in column "lb". For some instances, the solver was not able to optimally solve the LP relaxations, and for some others, no integer solution was found in $1 \mathrm{~h}$. In these cases, we cannot report the gap. Also, we do not report the solution time for the instances for which the solver ran out of memory. We observe here that even though the duality gaps are smaller compared to the ones of the AP instances, the first two formulations $S p H M P-B P-1$ and $S p H M P-B P-2$ perform poorly with large instances. On the contrary, the third formulation SpHMP-BP-3 is able to solve all instances to optimality in less than half an hour. The largest gap with SpHMP-BP-3 is 0.3\%. Even though larger percentages of variables are fixed with the randomly generated data, the effect of preprocessing on the solution time is not different compared to the AP data.

\section{Conclusion}

In this paper, we introduced two related star $p$-hub location problems, namely the Star $p$-hub Center Problem and the Star $p$-hub Median Problem with Bounded Path Lengths. We proposed two mixed integer programming formulations for the Star $p$-hub Center Problem and showed that, even though its LP-relaxation bound is very poor, the formulation with a smaller size is more efficient in solving our instances. For the Star $p$-hub Median Problem with Bounded Lengths, we proposed three integer programming formulations and then we strengthened the third one by the use of preprocessing. The third formulation uses specific clique inequalities and has much better performance than the first two ones. When strengthened by preprocessing, it enabled us to solve the considered instances within at most half an hour.

\section{Acknowledgments}

The research of the first author is supported by TUBITAK project no. 107M460.

\section{References}

[1] Alumur S, Kara BY. Network hub location problems: the state of the art. European Journal of Operational Research 2008;190:1-21.

[2] Alumur S, Kara BY, Karasan OE. The design of single allocation incomplete hub networks. Transportation Research Part B 2009;43:936-51.

[3] Campbell JF. Integer programming formulations of discrete hub location problems. European Journal of Operational Research 1994;72:387-405.

[4] Campbell JF. Hub location for time definite transportation. Computers and Operations Research 2009;36:3107-16.

[5] Campbell JF, Ernst AT, Krishnamoorthy M. Hub location problems. In: Drezner Z, Hamacher HW, editors. Facility location: applications and theory. Berlin: Springer; 2002. p. 373-407.

[6] Chardaire P, Lutton JL, Sutter A. Upper and lower bounds for the two-level simple plant location problem. Annals of Operations Research 1999;86: 117-40.

[7] Contreras I, Fernández E, Marín A. Tight bounds from a path based formulation for the tree of hub location problem. Computers and Operations Research 2009;36:3117-27.

[8] Contreras I, Fernández E, Marín A. The tree of hubs location problem. European Journal of Operational Research 2010;202:390-400.

[9] Cornuéjols G, Nemhauser GL, Wolsey LA. The uncapacitated facility location problem. In: Mirchandani PB, Francis RL, editors. Discrete location theory. New York: Wiley; 1990. p. 119-71.

[10] Cornuéjols G, Sridharan R, Thizy JM. A comparison of heuristics and relaxations for the capacitated plant location problem. European Journal of Operational Research 1991;50:280-97.

[11] Correia I, Nickel S, Saldanha-da-Gama F. The capacitated single-allocation hub location problem revisited: a note on a classical formulation. European Journal of Operational Research 2010;207:92-6.

[12] Ebery J. Solving large single allocation $p$-hub problems with two or three hubs. European Journal of Operational Research 2001;128:447-58.

[13] Ernst AT, Krishnamoorthy M. Efficient algorithms for the uncapacitated single allocation $p$-hub median problem. Location Science 1996;4:139-54.

[14] Ernst AT, Krishnamoorthy M. An exact solution approach based on shortest paths for $p$-hub median problems. INFORMS Journal on Computing 1998;10: 149-62.

[15] Ernst AT, Hamacher HW, Jiang H, Krishnamoorthy M, Woeginger G. Uncapacitated single and multi allocation $p$-hub center problems. Computers and Operations Research 2009;36:2230-41.

[16] Ernst AT, Jiang H, Krishnamoorthy M. Reformulations and computational results for uncapacitated single and multiple allocation hub covering problems. Unpublished report, CSIRO mathematical and information sciences, Australia; 2005.

[17] Gavish B. Topological design of centralized computer networks: formulations and algorithms. Networks 1982:12:355-77.

[19] Hamacher HW, Meyer T. Hub cover and hub center problems. Technical report, University of Kaiserslautern, Report in Wirtschaftsmathematik Nr. 98/ 2006.

[20] Helme MP, Magnanti TL. Designing satellite communication networks by zero-one quadratic programming. Networks 1989;19:427-50.

[21] Juette S, Gavriliouk EO, Hamacher HW. Polyhedral analysis of uncapacitated single allocation $p$-hub center problems. University of Kaiserslautern, Department of Mathematics, Report in Wirtschaftsmathematik Nr. 109, 2007.

[22] Kara BY, Tansel BC. On the single assignment p-hub center problem. European Journal of Operational Research 2000;125:648-55.

[23] Kara BY, Tansel BC. The single assignment hub covering problem: models and linearizations. Journal of the Operational Research Society 2003;54:59-64.

[24] Kariv O, Hakimi SL. An algorithmic approach to network location problems. I: the p-centers. SIAM Journal on Applied Mathematics 1979;37:513-38.

[25] Krarup J, Pruzan PM. The simple plant location problem: survey and synthesis. European Journal of Operational Research 1983;12:36-81.

[26] Labbé M, Peeters D, Thisse JF. Location on networks. In: Ball MO, Magnanti TL Monma CL, Nemhauser GL, editors. Network routing, handbooks in operations research and management sciences, vol. 8. North-Holland, Amsterdam, Elsevier; 1995. p. 551-624.

[27] Labbé M, Yaman H. Projecting the flow variables for hub location problems. Networks 2004;44:84-93. 
[28] Labbé M, Yaman H. Solving the hub location problem in a star/star network. Networks 2008;51:19-33.

[29] Labbé M, Yaman H, Gourdin E. A branch and cut algorithm for hub location problems with single assignment. Mathematical Programming 2005;102: 371-405.

[30] Meyer T, Ernst AT, Krishnamoorthy M. A 2-phase algorithm for solving the single allocation $p$-hub center problem. Computers and Operations Research 2009;36:3143-51.

[31] O'Kelly ME. A quadratic integer program for the location of interacting hub facilities. European Journal of Operational Research 1987;32:393-404.

[32] O'Kelly ME, Miller HJ. Solution strategies for the single facility minimax hub location problem. Papers in Regional Science 1991;70:367-80.

[33] Pamuk FS, Sepil C. A solution to the hub center problem via a singlerelocation algorithm with tabu search. IIE Transactions 2001;33:399-411.

[34] Pirkul H, Schilling D. An efficient procedure for designing single allocation hub and spoke systems. Management Science 1998;44:S235-42.

[35] Skorin-Kapov D, Skorin-Kapov J, O'Kelly M. Tight linear programming relaxations of uncapacitated $p$-hub median problem. European Journal of Operational Research 1996;94:582-93.
[36] Sohn J, Park S. A linear program for the two hub location problem. European Journal of Operational Research 1997;100:617-22.

[37] Sridharan R. The capacitated plant location problem. European Journal of Operational Research 1995;87:203-13.

[38] Wagner B. Model formulations for hub covering problems. Journal of the Operational Research Society 2008;59:932-8.

[39] Yaman H. Star p-hub median problem with modular arc capacities. Computers and Operations Research 2008;35:3009-19.

[40] Yaman H. The hierarchical hub median problem with single assignment. Transportation Research Part B 2009;43:643-58.

[41] Yaman H. Allocation strategies in hub networks. European Journal of Operational Research 2011;211:442-51.

[42] Yaman H, Kara BY, Tansel BC. The latest arrival hub location problem for cargo delivery systems with stopovers. Transportation Research B 2007;41: 906-19.

[43] Yaman H, Karasan OE, Kara BY. Release time scheduling and hub location for next day delivery. Technical report, Bilkent University, Department of Industrial Engineering; 2010. 